\title{
Pathways to Health Services Utilization: Overcoming Economic Barriers through Support Mechanisms
}

\author{
Priscila Diaz ${ }^{1}$, Jessica Stahl², David Lovis-McMahon ${ }^{2}$, Summer H. Y. Kim², \\ Virginia S. Y. Kwan ${ }^{2}$ \\ ${ }^{1}$ Psychology Department, Azusa Pacific University, Azusa, USA \\ ${ }^{2}$ Psychology Department, Arizona State University, Tempe, USA \\ Email:pdiaz@apu.edu
}

Received June $12^{\text {th }}, 2013$; revised July $12^{\text {th }}, 2013$; accepted July $19^{\text {th }}, 2013$

Copyright (C) 2013 Priscila Dia et al. This is an open access article distributed under the Creative Commons Attribution License, which permits unrestricted use, distribution, and reproduction in any medium, provided the original work is properly cited.

\begin{abstract}
Objective: By 2014, over 30 million Americans currently lacking health insurance will be able to access health services. While enhancing accessibility to healthcare is a significant step towards reducing health disparities, it is unclear whether access to health services will result in utilization of such services. Previous studies demonstrate that lower socioeconomic status (SES) is a key social determinant of poor health outcomes. The present research examined the potential SES gradient in the utilization of medical services considering healthcare accessibility, and how family support influences healthcare decision-making. Methods: A sample of young Americans with universal access to healthcare services was surveyed to determine whether decisions to visit the doctor for certain symptoms are differed by SES. In a follow-up young adult sample, the bearing of various forms of family support was examined as potential mechanisms that may explain SES differences in seeking healthcare. Results: Among informational, financial, and emotional support, it was found that emotional (how emotionally supportive family is when one is ill) and informational support (how often one consults family for health-related issues) mediated SES and the frequency of health services utilization. Higher SES individuals received more emotional and informational health support from family, which was associated with more immediate healthcare utilization. Conclusion: The findings suggest an explanation for incrementally worse health outcomes for lower SES individuals because of their delay in seeking medical attention. While SES is not a controllable factor, strengthening support networks for health consultation has valuable implications for healthcare promotion and management.
\end{abstract}

Keywords: Health Services Utilization; Healthcare Coverage; Socioeconomic Status; Social Support

\section{Introduction}

Health disparities are defined as differences in disease prevalence, health status or access to healthcare based on population demographics US Department of Health and Human Services, 2011. Currently, uninsured Americans have the poorest health outcomes and many are from low socioeconomic backgrounds (DeNavas-Walt, Proctor, \& Smith, 2007). To address long-standing health disparities in the US, President Obama signed into law the Patient Protection and Affordable Care Act (PPACA, March 23rd, 2010) that proposes access to healthcare by 2014 for an estimated 30 million Americans who are currently uninsured (Consumer Health Reports, 2010). Specifically, PPACA allocates government subsidies to increase accessibility of health services for individuals with low socioeconomic status (SES).

Healthcare reform has generated a contentious political arena beginning with early legal challenges from several states, declaring the law unconstitutional and leading to a recent repeal of PPACA (Davis \& Litvan, 2011). Despite the political maelstrom surrounding healthcare reform, public opinion is clearly divided, vacillating between support and opposition over time
(Brodie, Altman, Deane, Buscho, \& Hamel, 2010). Vice President Biden expressed his optimism in 2010 as PPACA was being signed into law at The White House: "Mr. President, you are... literally about to make history... tens of millions of Americans will be a whole lot healthier from this moment on." While enhancing accessibility to healthcare is a significant step forward, little is known about whether merely having coverage for health services will actually lead to timely, preventative utilization of health services.

Lack of adequate healthcare coverage has often been noted as a major cause of health disparities in the United States. Nevertheless, health disparities persist even in countries with universal healthcare such as England (Townsend \& Davidson, 1982) and Sweden (Lundberg, 1991). Socioeconomic statuswhere an individual stands in society in terms of income, education, and occupational prestige - is a consistent predictor of health outcomes across samples of different demographics. The pattern is a gradient such that, with every incremental decrease in socioeconomic status, health status also worsens incrementally with lower SES individuals reporting worse health status (Adler, Boyce, Chesney, Folkman, \& Kahn et al., 1993). This gradient has been widely documented across ethnic groups, sex, 
occupational backgrounds, age groups, as well as in other representative samples in the US (e.g., Ostrove, Feldman, \& Adler, 1999; Goodman, Adler, Daniels, Morrison, Slap, \& Dolan, 2003; Goodman, McEwen, Dolan, Schafer-Kalkhoff, \& Adler, 2005; Operario, Adler, \& Williams, 2004).

The bulk of literature regarding the SES-health gradient clearly demonstrates that SES predicts health status regardless of whether the individual has access to healthcare. Why do SES differences in health status persist even when individuals have equal access to medical services? Are there behavioral differences in medical attention seeking related with SES? Past literature has focused on actual health outcomes and has not addressed how SES relates to healthcare utilization. Understanding SES differences in healthcare utilization may provide insight into inclusive approaches towards reducing health disparities. Healthcare utilization refers to a variety of medical attention seeking behaviors including going to the doctor (Gorman \& Braverman, 2008). Different socioeconomic groups may exhibit varying levels of healthcare utilization even when they all have access to health services, which may provide insight into the SES gradient regarding health outcomes.

A major aim of this research is to extend the work regarding the SES-health status gradient to include the concept of healthcare utilization. SES is expected to be a significant determinant of utilization when access to healthcare is not a limiting factor. In two studies, we recruited an all-insured sample that sufficiently varies in SES but equivalent in access to healthcare service, of similar age, and comparable levels of education. Rather than recruiting in the general community where health insurance policies differ between individuals and healthcare coverage varies by SES, the type of insurance coverage was equivalent across all participants in this study (i.e. university students), even of different SES backgrounds. This sample mirrors what PPACA aims to accomplish in the general population, and therefore helps to simulate what might occur when most Americans obtain reasonable access to health insurance.

In Study 1, we predicted individuals from lower socioeconomic groups to utilize healthcare services for common symptoms less frequently than individuals from higher socioeconomic groups. Another major aim of this research investigates what factors may facilitate or hinder healthcare utilization service among young adults from low SES. Study 2 examines three forms of health related family support that account for differences in healthcare utilization by SES, which is suggested by the bodies of literature on socialization of health behaviors and on forms of social support. By identifying these psychosocial factors, intervention programs may be structured to promote timely utilization of health services and the effectiveness of US healthcare.

\section{Study 1: Healthcare Utilization and the SES Gradient}

\section{Method}

Participants. There were 194 individuals (mean age $=19$ ) who indicated having student healthcare coverage (female = 111). The insurance co-pay for a health visit was $\$ 10$. Ethical guidelines were followed and the study was approved by the Institutional Review Board Committee. The participants attended a southwestern state university and were compensated with 1 credit hour to complete a research requirement. The sample consisted of 153 White/Caucasian, 36 Latino/Hispanic, 4 indicated other. In validating the data, sixteen participants were excluded. Eleven were excluded because they stated that they did not have insurance. The remaining five were excluded because they had missing values for either the SES or the Healthcare Utilization questions.

Materials. To assess when individuals make the decision to see a physician about an illness, participants were asked to choose one of five answer choices that increased in severity or duration for six common health symptoms (i.e., cough, stomach pain, heartburn, dizziness, sore throat, and fever). Response values depended on the symptom; for example, the symptom Cough included: 1 = One week, $2=$ Two weeks, $3=$ One month, $4=$ Two months, $5=$ Three months, $6=$ would not go to the doctor until symptom lasted longer; whereas the symptom Fever included: $1=100^{\circ}, 2=101^{\circ}, 3=102^{\circ}, 4=103^{\circ}, 5=104^{\circ}, 6$ $=$ would not go to the doctor until symptom got worse. Participants were asked to recall their prior experience to answer the questions or to make a best guess about what they would do if they did have the symptom. The above were chosen based on a pool of common symptoms listed on MayoClinic.com and their generalizability for young adults, since it is likely that one has experienced at least a few of the above symptoms by age 18 . The mean response to the healthcare utilization questions was calculated for each participant. This healthcare utilization index had a minimum score of 1.17 and a maximum score of 5.33 . (Cronbach's alpha $=.64$ ).

To examine the SES-health gradient, we measured both objective SES (e.g., education attainment, household income, occupation type, and wealth), and subjective SES (e.g., MacArthur Scale of Subjective Social Status, which asks participants to indicate their position in a 9- or 10-rung ladder where the top of the ladder indicated highest SES and bottom indicated lowest SES). While both objective and subjective measures independently predict health outcomes, subjective SES captures finer gradations of objective indicators, such that it reflects the difference in quality (e.g., quality of education based on the prestige of the school) that is not captured by the objective indicators. To determine subjective SES, we asked participants to choose their family's socioeconomic status in terms of income (working class $=1$ to upper class $=5$ ). The majority of participants reported middle class $(\mathrm{M}=3.40, \mathrm{SD}$ $=.85)$.

\section{Results}

To examine how individuals of varying levels of SES utilize healthcare for several symptoms, a bivariate correlation between SES and health utilization was conducted. SES and healthcare utilization were significantly related, $\mathrm{r}(178)=-.17$, $p<.05$, indicating that individuals from a lower SES background delay utilizing healthcare for various symptoms (see Figure 1).

The results correspond to the gradient in health outcomes based on SES as documented in past literature, but extend this work to include the utilization of healthcare services. Findings of Study 1 document an SES difference in health services utilization; individuals from lower socioeconomic status utilize health services less frequently than their higher SES counterparts. Every participant had access to an insurance policy, so availability of access could not explain the pattern of utilization. The link between SES and utilization of healthcare may con- 


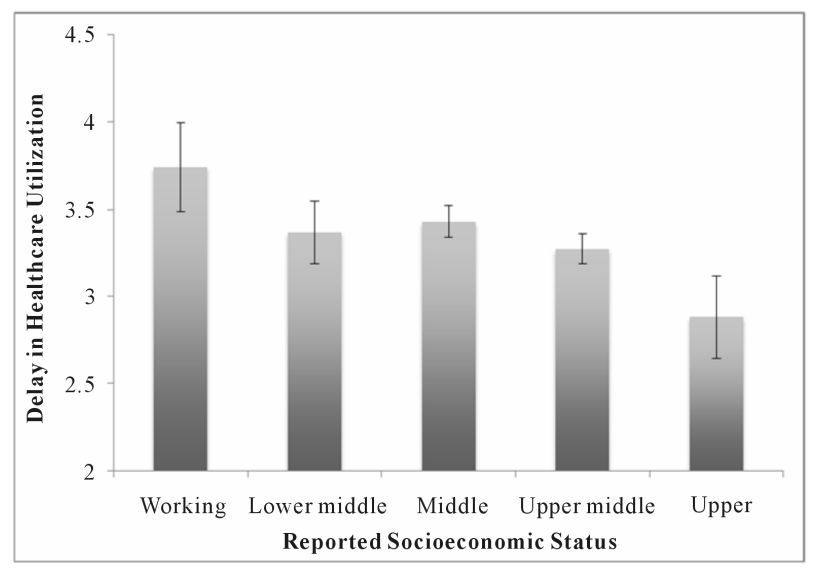

Figure 1.

Socioeconomic status gradient in the delay of utilizing healthcare services.

tribute to the SES-health gradient considering that individuals of lower SES are not seeking medical attention in a timely manner. Symptoms left untreated may lead to serious illness; hence, a follow-up study examined the support mechanisms involved in this process.

\section{Study 2: Contextual Factors between SES and Healthcare Utilization Link}

\section{Method}

Why did lower SES individuals with universal access to healthcare delay seeking medical attention? SES is a key social determinant of health that begins with delaying healthcare utilization as shown in Study 1. However, the specific mechanisms and processes of healthcare utilization remain unclear. What factors may influence healthcare utilization among individuals of different socioeconomic backgrounds? It may be that the action of utilizing healthcare is socialized within families from an early age and sustained through a supportive network, much like the formation of our attitudes, behaviors, and habits. Thus, three forms of family support (emotional, informational, financial) were investigated as intermediary links between SES and healthcare utilization.

Families are the basic foundation for social connectedness and supportive relationships. Social connectedness creates a web of support that builds norms for adopting healthy habits, diffuses information about health, and promotes access to services (Kawachi, Kennedy, \& Glass, 1999). Individuals who decide to seek help from their social network are also more likely to receive recommendations for assistance (Dew, Bromet, Schulberg, \& Parkinson, 1991; Vogel, Wade, Wester, Larson, \& Hackler, 2007).

Prior research demonstrates the persistent influence of parental socialization on health beliefs, even after children become young adults and leave home (e.g., to attend college; Lau, Quadrel, \& Hartman, 1990). The weight of socialization, attitude internalization and belief formation from the family may shape behaviors toward medical decisions, health-related personnel, and health services utilization patterns. More fundamentally, children learn from their parents to identify and define their bodily feelings as symptoms that may or may not need medical attention (Cardol, Groenewegen, Spreeuwenberg,
Van Dijk, Van Den Bosch, \& De Bakker, 2006). As a result of this socialization process, family members utilize medical services in similar ways.

In addition, previous research shows that when we experience the symptoms of an illness, we first seek help from our social network (e.g., family) before seeking professional help (Horwitz, 1977). The types of help that we seek from our social network can be categorized into different types of social support: emotional support and instrumental support (Seeman, 1996; House, Umberson, \& Landis, 1988). Emotional support includes providing encouragement and a sense of comfort. Instrumental support may be organized into further subcategories, such as financial support (providing monetary help) and informational support (providing advice). Given that social supportive connections play a vital role in an individual's decisions (including health behaviors), we posited that one's family members would be a source of support and that such support would positively relate to health outcomes. Consequently, Study 2 investigated the pattern of three distinct means of family social support (i.e., emotional, informational and financial support) on healthcare utilization in consideration of SES. Increased levels of these three support mechanisms were predicted to mediate the relationship between SES and the decision of when to see the doctor.

\section{Method}

Participants. There were 557 students (female $=308)$ with health insurance attending a southwestern state university (mean age $=19.6$ ). The insurance co-pay for a health visit was $\$ 10$. The study was approved by the Institutional Review Board Committee and ethical guidelines with participants were followed. The sample consisted of 356 White/Caucasian, $87 \mathrm{La}-$ tino/Hispanic, 30 Black/African American, 8 Native American, 41 Asian/Asian-American, 14 Arab/Middle Eastern and 20 indicated Other. Of the initial 557 participants, forty-eight were excluded for stating that they did not have health insurance. A further thirteen participants were excluded for having some combination of missing values on the SES, three family support items, or the healthcare utilization index.

Materials. Healthcare utilization and SES measures from Study 1 were used in Study 2. As with the Study 1, each participant received a healthcare utilization index score. This healthcare utilization index had a minimum score of 1.17 and a maximum score of 5.83. Cronbach's alpha for the index was .69. The majority of participants reported middle class $(\mathrm{M}=3.30$, $\mathrm{SD}=.86)$.

Three items assessed the type and level of family support with an illness. One item asked participants to report how emotionally supportive their family would be if they were sick and the second item asked about financial support $(1=$ Not supportive to $5=$ Very supportive). The third item assessed the level of communication about health within one's family by asking participants if they consulted with their family about medical decisions $(1=$ Very often to $5=$ Never $)$.

\section{Results}

Bivariate correlations were conducted to examine the relationships between SES, family support factors and health utilization (see Table 1). Similar to Study 1 results, SES was significantly related to healthcare utilization $(\mathrm{r}=-.10, p<.05)$. 
Table 1.

Study 2 correlations between socioeconomic status, support and healthcare utilization behaviors.

\begin{tabular}{lccccc}
\hline & 1 & 2 & 3 & 4 & 5 \\
\hline 1. SES & 1 & $.214^{* *}$ & $.109^{*}$ & $.323^{* *}$ & $-.095^{*}$ \\
2. Information & & 1 & $.243^{* *}$ & $.301^{* *}$ & $-.257^{* *}$ \\
3. Emotional & & & 1 & $.430^{* *}$ & $-.156^{* *}$ \\
$\begin{array}{l}\text { 4. Financial } \\
\text { 5. Healthcare }\end{array} \quad$ & & & 1 & -.081 \\
$\quad$ Utilization & & & & & 1 \\
\hline
\end{tabular}

Individuals who reported a lower SES tended to wait longer to visit the doctor. SES also correlated with emotional support, $(\mathrm{r}=.11, p<.05)$, financial support, $(\mathrm{r}=.32, p<.05)$, and informational support, $(\mathrm{r}=.22, p<.05)$. Individuals who reported a higher SES generally communicated with their family about healthcare to a greater extent and reported greater emotional and financial support. However, healthcare utilization was significantly related to emotional support $(\mathrm{r}=-.26, p<.05)$ and informational support $(\mathrm{r}=-.16, p<.05)$ but not financial support $(\mathrm{r}=-.08, p>.05)$. With greater emotional and informational support, individuals with health insurance were more likely to visit the doctor sooner.

Based on the results of the correlation analyses, we next tested whether informational support and emotional support are significant mediators underlying the effects of SES on healthcare utilization. Following Baron and Kenny's (1986) four-step procedure, we conducted a series of regression analyses to establish the mediating role of information support. On step 1, SES was significantly associated with informational support ( $\beta$ $\left.=.21 ; \mathrm{R}^{2}=.05, p<.01\right)$. On step 2 , SES was significantly associated with healthcare utilization $\left(\beta=-.10 ; \mathrm{R}^{2}=.01, p<.05\right)$. On step 3, informational support was significantly associated with healthcare utilization $\left(\beta=-.26 ; \mathrm{R}^{2}=.07, p<.01\right)$. On step 4 , the regression of healthcare utilization on informational support $(\beta=-.25, p<.05)$ was significant but not SES $(\beta=-.04, p$ $>.05$ ) with the overall model $\mathrm{R}^{2}=.07, p<.01$. The relationship between SES and healthcare utilization did not remain significant when family informational support is accounted for, suggesting that the relationship between SES and healthcare utilization is mediated by informational support (Sobel test, $\mathrm{z}=$ $-3.76, p<.01)$. Thus, individuals with higher SES received more health consultation from family, which, in turn, associated with more immediate healthcare utilization (Figure 2).

Likewise, we conducted a series of regression analyses to establish the mediating role of emotional support. On step 1, SES was significantly associated with emotional support $(\beta$ $\left.=.11 ; \mathrm{R}^{2}=.01, p<.05\right)$. On step 2 , SES was significantly associated with healthcare utilization $\left(\beta=-.10 ; \mathrm{R}^{2}=.01, p<.05\right)$. On step 3, emotional support was significantly associated with healthcare utilization $\left(\beta=-.15 ; \mathrm{R}^{2}=.02, p<.05\right)$. On step 4 , the regression of healthcare utilization on emotional support $(\beta$ $=-.15, p<.05)$ was significant but not for SES $(\beta=-.08, p$ $>.05$ ) with the overall model $\mathrm{R}^{2}=.04, p<.05$. The relationship between SES and healthcare utilization did not remain significant with family emotional support, suggesting that the relationship between SES and healthcare utilization is mediated by emotional support (Sobel test, $\mathrm{z}=-1.99, p<.05$ ). Thus, individuals with higher SES tended to receive more emotional health support from family, which, in turn, was associated with more immediate healthcare utilization (Figure 3). Together, these findings provide mechanisms of the contextual factors that may contribute to the effect of SES on healthcare utilization. In particular, health information and emotional support provided by family are strong determinants in explaining how individuals from varying levels of SES differ in their healthcare utilization.

\section{Conclusion}

The current findings demonstrate that in a sample with universal access to healthcare, low SES individuals tend to delay utilization of healthcare when presented with concerning symptoms (i.e., fever of $104^{\circ}$ ). Family emotional and informational support accounts for these differences in SES and healthcare utilization behaviors. The landmark Whitehall studies (Marmot, Stansfield, Patel, North, Head, White et al., 1991) identified an SES gradient in health outcomes among workers with universal access to health services, in which lower SES individuals had incrementally worse health outcomes at every level of the gradient. Study 1 revealed a SES-utilization gradient with individuals from lower SES backgrounds delaying utilization of health services for various symptoms. Despite concerns about newly insured patients overwhelming the healthcare system (Heflin, 2010), access does not necessarily lead to greater utilization as Study 1 and 2 findings show low SES individuals delay medical attention. These findings suggest a potential explanation for incrementally worse health outcomes for lower SES individuals because of their delay in

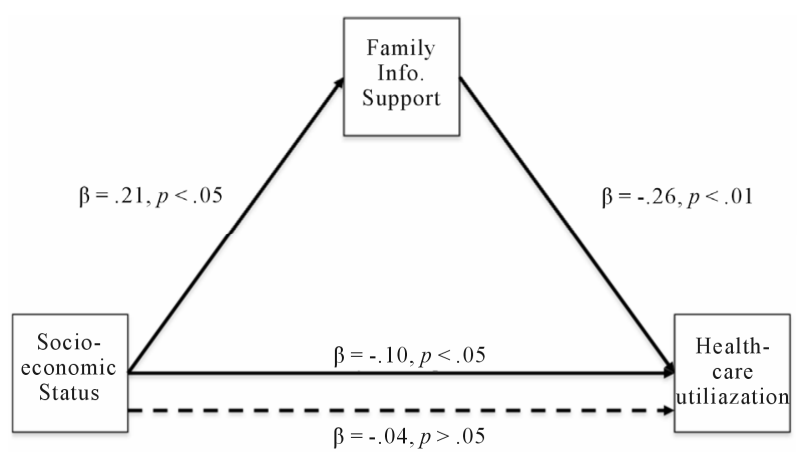

Figure 2.

The mediation of socioeconomic status on the delay of utilizing healthcare services by Family Informational Support.

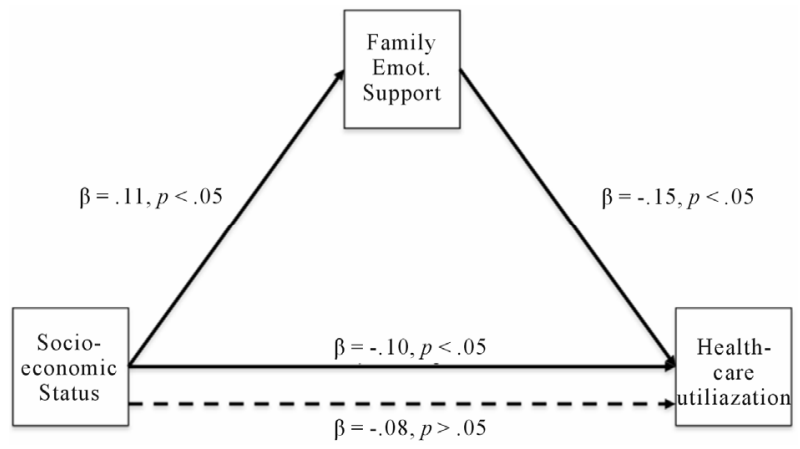

Figure 3.

The partial mediation of socioeconomic status on the delay of utilizing healthcare services by Family Emotional Support. 
seeking medical attention. Identifying the key factors concomitant with timely, appropriate health services utilization could significantly increase the effectiveness of health promotion efforts.

Prior research has established that family does play a part in how individuals seek professional help. Similarly, Study 2 found that higher SES individuals report greater emotional and financial support and communicated with their family about healthcare to a greater extent. The SES-utilization gradient from Study 1 was replicated as lower SES individuals waited longer to visit the doctor for symptoms. The key to understanding the SES-utilization gradient may lie in the fact that individuals with higher SES engaged in more health consultation with their family and received greater emotional support. These two factors associated with timely healthcare utilization, whereas financial support was not significantly related to health services utilization among individuals with health insurance. Thus, the relationship between SES, emotional support and health consultation with close others may be a key factor in medical attention seeking behavior. In that case, health promotion efforts focused on increasing communication about health matters with family, friends, and significant others may be highly effective, which would be congruent with the success of peer mentor programs, and community health advocates.

The generalization of the results is limited to college students, which may suitable in this initial investigation. Given that the U.S. currently does not have universal healthcare and that all provisions of the PPACA will not be fully implemented until 2018 , it is challenging to select a sample with sufficient diversity and universal access to health services. In that sense, the sample was both demographically representative and uniquely homogenous with regard to access to health services since all students had access to the same student health insurance and the student health center, which was conveniently located on the campus. Thus, universal access to care was removed as an obstacle for this study whereas it remains a challenge in the US population. Future research should include a larger sample with more diversity in terms of age, level of education, occupations, health status, and life experience. It would likely be informative to explore the specific content and characteristics of emotional support and health consultation with significant others that lead to health service utilization, particularly for groups where family dependence varies. Certain trusted figures may have more influence than others (e.g., mother vs. best friend) and possibly more frequent discussions with multiple people have a more powerful influence on health decision-making. Beyond the scope of the current research is to evaluate the appropriate time to utilize health services. Future studies should incorporate doctor's recommendations on when to seek medical attention for these symptoms (a fever of $100^{\circ}$ vs. a fever of $104^{\circ}$ ), in order to accurately utilize healthcare services when given access and survey individuals' real experiences with these illnesses rather than hypothetical accounts.

Public health campaigns, health directives from medical professionals, and community health efforts all seek to increase positive health outcomes. Yet, it has been challenging to identify the major characteristics of successful health promotion. Society has generally relied upon retrospective analyses to determine the effectiveness of health promotion efforts rather than being able to confidently predict whether they will be effective. Much of the recent debate regarding healthcare reform has focused on the need to reduce utilization of health services because of the perceived costs and the anticipated strain on the current medical system when 32 million Americans begin to utilize health services (Cutler, 2010). However, the preponderance of research elucidates the high financial and social costs of ineffective health promotion efforts and the negative, long-term health effects of delayed healthcare, emphasizing the benefits of timely health services utilization. This research demonstrates that supportive emotional relationships and health consultation with significant others is an important aspect of individuals not delaying medical attention. This provides an operative framework for appropriate public health messages, programs, and campaigns. It could also provide health professionals with a constructive avenue to conversations with patients regarding health issues as they encourage the patient to share information with their significant others rather than focusing solely on conveying health information to the patient in the medical setting. Effective health promotion efforts may also be the soundest way to reduce the prevalence of diseases (e.g., obesity, diabetes) that are rooted in lifestyle choices wherein the patient does not perceive the necessity for behavior change. This research suggests that individuals who share, trust and seek advice on health issues with significant others are more likely to utilize health services, which also suggests that they take a more proactive approach towards their own health. Successful health promotion efforts are essential as society faces an unprecedented urgency for effective health promotion methods with millions of new patients entering into the healthcare system.

\section{Acknowledgements}

We would like to thank the following individuals for their comments on an earlier version of this manuscript and help with data collection: Andrea Fessler, Aresh Vasefi, Benjamin Lozada, Jason Baxter, Jose Alba and Megan Leonhardt. The first author has been partially supported by National Institute of Mental Health Grant T32MH018387-24.

\section{REFERENCES}

Adler, N. E., Boyce, T., Chesney, M. A., Cohen, S., Folkman, S., Kahn, R. L., \& Syme, S. L. (1994). Socioeconomic status and health: The challenge of the gradient. American Psychologist, 49, 15-24. doi: 10.1037/0003-066X.49.1.15

Baron, R. M., \& Kenny, D. A. (1986). The moderator-mediator variable distinction in social psychological research: Conceptual, strategic, and statistical considerations. Journal of Personality and Social Psychology, 51, 1173-1182. doi:10.1037/0022-3514.51.6.1173

Brodie, M., Altman, D., Deane, C., Buscho S., \& Hamel, E. (2010). Liking the pieces, not the package: Contradictions in public opinion during health reform. Health Affairs, 29, 2323-2334. doi: $10.1377 /$ hlthaff.2010.0434

Cardol, M., Groenewegen, P. P., Spreeuwenberg, P., Van Dijk, L., Van Den Bosch, W. J. H. M., \& De Bakker, D. H. (2006). Why does it run in families? Explaining family similarity in help-seeking behaviour by shared circumstances, socialization, and selection. Social Science \& Medicine, 63, 920-932. doi:10.1016/j.socscimed.2006.01.025

Consumer Health Reports (2010).

http://www.consumerreports.org/health/insurance/health-insurance/n ew-health-insurance-basics/uninsured.htm

Cutler, D. (2010). How health care reform must bend the cost curve. Health Affairs, 29, 1131-1135. doi:10.1377/hlthaff.2010.0416

Davis, J. H., \& Litvan, L. (2011). Senate republicans introduce healthoverhaul repeal. Bloomber Business Week. http://www.bloomberg.com/news/2011-02-01/mcconnell-will-introd uce-senate-measure-to-repeal-obama-s-health-care-law.html 
DeNavas-Walt, C., Proctor, B. D., \& Smith, J. (2007). Income, poverty, and health insurance coverage in the United States: 2006. Washington DC: US Government Printing Office.

Dew, M. A., Bromet, E. J., Schulberg, H. C., \& Parkinson, D. K. (1991). Factors affecting service utilization for depression in a white collar population. Social Psychiatry and Psychiatric Epidemiology, 26, 230237. doi:10.1007/BF00788971

Goodman, E., Adler, N. E., Daniels, S. R., Morrison, J. A., Slap, G. B., \& Dolan, L. M. (2003). Impact of objective and subjective social status on obesity in a biracial cohort of adolescents. Obesity Research, 11, 1018-1026. doi:10.1038/oby.2003.140

Goodman, E., McEwen, B. S., Dolan, L. M., Schafer-Kalkhoff, T., \& Adler, N. E. (2005). Social disadvantage and adolescent stress. Journal of Adolescent Health, 37, 484-492.

doi:10.1016/j.jadohealth.2004.11.126

Gorman, B., \& Braverman, J. (2008). Family structure differences in healthcare utilization among U.S. children. Social Science \& Medicine, 67, 1766-1775. doi:10.1016/j.socscimed.2008.09.034

Heflin, J. (2010). Health reform threatens to cram already overwhelmed emergency rooms. The Hill.

http://thehill.com/business-a-lobbying/98025-health-reform-threatens -to-overwhelm-already-crammed-emergency-rooms

Horwitz, A. (1977). Pathways into psychiatric treatment: Some differences between men and women. Journal of Health and Social Behavior, 18, 169-178. doi:10.2307/2955380

House, J., Landis, K., \& Unberson, D. (1988). Social relationships and health. Science, 241, 540-545. doi:10.1126/science.3399889

Kawachi, I., Kennedy, B. P., \& Glass, R. (1999). Social capital and self-rated health: A contextual analysis. American Journal of Public Health, 89, 1187-1193. doi:10.2105/AJPH.89.8.1187
Lau, R., Quadrel, M., \& Hartman, K. (1990). Development and change of young adults' preventive health beliefs and behavior: Influence from parents and peers. Journal of Health and Social Behavior, 31, 240-259. doi: $10.2307 / 2136890$

Lundberg, O. (1991). Causal explanations to class inequalities in health: An empirical analysis. Social Science \& Medicine, 32, 385-394. doi:10.1016/0277-9536(91)90339-E

Marmot, M. G., Stansfield, S., Patel, C., North, F., Head, J., White, I., Brunner, E., Feeney, A., Marmot, M. G., \& Davey-Smith, G. (1991). Health inequalities among British civil servants: The whitehall II study. Lancet, 337, 1387-1393. doi:10.1016/0140-6736(91)93068-K

Operario, D., Adler, N. E., \& Williams, D. R. (2004). Subjective social status: Reliability and predictive utility for global health. Psychology \& Health, 19, 237-246. doi:10.1080/08870440310001638098

Ostrove, J. M., Feldman, P., \& Adler, N. E. (1999). Relations among socioeconomic status indicators and health for African-Americans and Whites. Journal of Health Psychology, 4, 451-463. doi:10.1177/135910539900400401

Patient Protection and Affordable Care Act (2010). PPACA, P.L., 23 March 2010, 111-148.

Seeman, T. E. (1996). Social ties and health. Annals of Epidemiology, 6, 442-451. doi:10.1016/S1047-2797(96)00095-6

Townsend, P., \& Davidson, N. (1982). Inequalities in health: The black report. Penguin: Harmondsworth.

US Department of Health and Human Services (2011). http:/www.hhs.gov/news/press/2011pres/04/04hdplan04082011.htm Vogel, D. L., Wade, N. G., Wester, S. R., Larson, L., \& Hackler, A. H. (2007). Seeking help from a mental health professional: The influence of one's social network. Journal of Clinical Psychology, 63, 233-245. doi:10.1002/jclp.20345 